University of South Carolina

Scholar Commons

$10-30-2013$

Contributions to Pion Decay from Lorentz Violation in the Weak Sector

Brett David Altschul

altschul@mailbox.sc.edu

Follow this and additional works at: https://scholarcommons.sc.edu/phys_facpub

Part of the Physics Commons

Publication Info

Published in Physical Review D, Volume 88, Issue 7, 2013.

(C) 2013 American Physical Society

This Article is brought to you by the Physics and Astronomy, Department of at Scholar Commons. It has been accepted for inclusion in Faculty Publications by an authorized administrator of Scholar Commons. For more information, please contact digres@mailbox.sc.edu. 


\title{
Contributions to Pion Decay from Lorentz Violation in the Weak Sector
}

\author{
Brett Altschul] \\ Department of Physics and Astronomy \\ University of South Carolina \\ Columbia, SC 29208
}

\begin{abstract}
Lorentz violation in the weak sector would affect the $\beta$-decay lifetimes of pions. The decay amplitude may be rendered anisotropic, but only an isotropic violation of boost invariance can affect the net lifetime in the center of mass frame. However, since the rest frames of the pions that produce the NuMI neutrino beam at Fermilab vary with the rotation of the Earth, it is possible to constrain anisotropic Lorentz violation using prior analyses of sidereal variations in the event rate at the MINOS near detector. The resulting bounds on weak-sector Lorentz violation are at the $10^{-4}$ level, a substantial improvement over previous results. The highly relativistic character of the pions involved is responsible for the improvement.
\end{abstract}

\footnotetext{
${ }^{1}$ baltschu@physics.sc.edu
} 


\section{Introduction}

There has recently been a significant degree of interest in the possibility that the fundamental Lorentz and CPT symmetries of the standard model might not hold exactly. There has not so far been any significant experimental indication that these symmetries are broken in nature. However, the possibility is interesting, because if such symmetry breaking did exist, it would be indicative of a completely new regime of fundamental physics.

Lorentz and CPT violation involving standard model quanta can be described using the machinery of effective field theory. The general field theory that describes these effects is known as the standard model extension (SME). It contains all possible translationinvariant but Lorentz-violating operators that may be constructed out of known standard model fields. The effects of the Lorentz-violating operators are parameterized by tensorvalued coefficients [1, 2]. If Lorentz symmetry is broken spontaneously, these are related to the vacuum expectation values of dynamical fields with tensor indices.

The minimal SME - a restricted version of the theory that contains only renormalizable operators - is now the standard framework used for describing the results of experimental tests of isotropy, boost invariance, and CPT symmetry. Important tests of these symmetries have included studies of matter-antimatter asymmetries for trapped charged particles [3, 4, 5] and bound state systems [6, 7], measurements of muon properties [8, 9], analyses of the behavior of spin-polarized matter [10], frequency standard comparisons [11, 12, 13, 14, Michelson-Morley experiments with cryogenic resonators [15, 16, 17], measurements of neutral meson oscillations [18, 19, 20, 21, 22], polarization measurements on the light from cosmological sources [23, 24], high-energy astrophysical tests [25, 26, 27, 28], precision tests of gravity [29, 30], and others. Up-to-date information about the status of the relevant constraints may be found collected in [31].

Motivated by the claim from OPERA [32] that neutrinos might have been moving faster than light, we previously looked at the effects of leptonic Lorentz violation on the pion decay rate. It turned out that the kind of modification to the neutrino dispersion relation that would have been necessary to explain the purported OPERA result would have also led to drastic modifications to the pion decays that produced the neutrinos and thus to the experiment's beam structure. More recently, there has been interest in constraining Lorentz violation in the weak sector using analyses of $\beta$-decays [33, 34]. Studies of the energetics of isotopes' $\beta$-decay endpoints can also provide sensitivity to other types of Lorentz violation [35].

This work is a generalization of our previous analyses [36, 37] of how Lorentz violation could affect leptonic meson decays, such as $\pi^{-} \rightarrow \mu^{-}+\bar{\nu}_{\mu}$. The previous work dealt with Lorentz violation for the second-generation fermions. Our new results account for the possibility that the $W$ propagator may also be modified by a Lorentz-violating background tensor $\chi^{\mu \nu}$. This provides an interesting way to constrain weak sector Lorentz violation, and it turns out that working with relativistic pions provides significant improvements in 
sensitivity relative to studying the decays of stationary particles.

The outline of this paper is as follows. The decay rate for a pion in the presence of weak-sector Lorentz violation is evaluated in section 2, In section 3, this result is combined with existing analyses of MINOS near detector data to place new constraints on the Lorentz violation coefficients involved. Section 4 discusses these result in context, including a critique of some previously claimed constraints on a number of the same coefficients. An appendix extends the calculations in section 2 to show how measuring particle spins could theoretically provide sensitivity to additinal coefficients, although this is experimentally impractical.

\section{Lorentz Violation in Pion Decay}

The present calculations will assume that Lorentz violation only exists in the pure weak and Higgs sectors. This means the tensor structure of the $W$ propagator will be modified, but the kinematics of the parent and daughter particles will not. The calculation may be carried out in the rest frame of the pion, and all the kinematical factor associated with both the incoming and outgoing particles are unchanged from the usual case. So only the modified matrix element needs to be calculated, and its modified form is actually quite simple. A similar calculation, but assuming a real, traceless $\chi$ that was isotropic in the laboratory frame, was performed in [38]. Naturally, since any real Lorentz violation is a fairly small effect, we may neglect all effects beyond leading order. The modifications uncovered in the present calculation may therefore simply be added to those found in [37] if there is relevant Lorentz violation in both the weak and muon sectors.

The specific form of Lorentz violation we shall consider is a CPT-even modification of the $W$ propagator. In the Feynman gauge, and in the limit in which the exchanged momentum is negligible compared to the $W$ boson mass $\left(\left|p^{2}\right| \ll m_{W}^{2}\right)$, the propagator becomes

$$
D^{\mu \nu}(p \rightarrow 0)=-i \frac{g^{\mu \nu}+\chi^{\mu \nu}}{-m_{W}^{2}} .
$$

The complex tensor $\chi^{\mu \nu}$ is an effective construction, related to Lorentz violation coefficients that arise in the pure gauge and Higgs boson sectors [39, 34]. In the present limit, with the exchanged $W$ far off shell, $\chi$ takes the form

$$
\chi^{\mu \nu}=-k_{\phi \phi}^{\mu \nu}-\frac{i}{2 g} k_{\phi W}^{\mu \nu}+\frac{2 p_{\alpha} p_{\beta}}{m_{W}^{2}} k_{W}^{\alpha \mu \beta \nu} .
$$

The tensors $k_{\phi \phi}, k_{\phi W}$, and $k_{W}$ describe CPT-even forms of Lorentz violation in the Lagrangian; they relate to the Higgs sector, to Higgs-weak mixing, and to the pure $W$ sector, respectively. After spontaneous symmetry breaking, when the Higgs field acquires a vacuum expectation value, coupling terms between the scalar field and the $W$ produce operators that are simply bilinear in the $W$ field and so contribute to the $W$ propagator. 
There are additional terms in this propagator related to each of the $k_{\phi \phi}, k_{\phi W}$, and $k_{W}$ tensors, but those appearing in (2) are the dominant contributions from each of those tensors; further terms are suppressed by relative powers of $p^{2} / m_{W}^{2}$. In the rest frame of a decaying pion, where the $W$ momentum is purely timelike, the momentum-dependent $k_{W}$ term in (2) becomes $2\left(m_{\pi}^{2} / m_{W}^{2}\right) k_{W}^{0 \mu 0 \nu}$. More generally, the structure of the $k_{W}$ contribution to the effective $\chi$ will depend on the experimental conditions, and so results from different decays will provide complementary sensitivities. However, since the firstorder dependence of $\chi$ on $k_{W}$ is comparable to higher-order corrections to the $k_{\phi \phi}$ and $k_{\phi W}$ dependences, we shall mostly treat experimental results pertaining to $\chi$ as providing constraints on the two-index tensors $k_{\phi \phi}$ and $k_{\phi W}$. Hermiticity ensures that the symmetric part of $\chi^{\mu \nu}$ is real and the antisymmetric part is purely imaginary. Consideration of isotropic, boost-invariance-violating $\chi$ terms dates back to [38, 40], but the analysis here will include anisotropic terms as well.

In general, calculations of scattering cross sections and decay rates for reactions with Lorentz violation can be tricky. The methods for performing such calculations were worked out in [41]. Kinematic modifications, which are determined by the energy-momentum relations for the incoming and outgoing particles, are often more important than any changes to the matrix element. However, the pion, muon, and neutrino dispersion relations are all conventional in this case, and so the calculations simplify considerably. All that is necessary is to compute the matrix element using the modified propagator (11) instead of the usual one, in the usual tree-level Feynman diagram that describes the pion decay.

In the rest frame of a decaying pion, the decay rate can only depend on isotropic forms of Lorentz violation - those that only violate Lorentz boost symmetry. The reason is that the unperturbed decay is isotropic in the center of mass frame. Effects that break rotation symmetry will produce a decay amplitude that depends on the momentum directions of the outgoing particles. However, when the decay rate is integrated over all angular channels, the anisotropies will cancel out; an increased rate for a daughter particle to be emitted in one direction will be canceled by a decreased rate for a decay along a different direction. In a general theory, it is difficult to demonstrate this fact explicitly; the angular dependence of the decay amplitude is quite complicated. However, when the only source of Lorentz violation for this process is in the weak sector, the calculation of the angular dependence is simple enough to done explicitly. We shall therefore include all forms of isotropy breaking in our present calculations, with the expectation that they will all cancel out in the final expression for the pion decay rate. The tensor $\chi^{\mu \nu}$ consists of a symmetric real part and an antisymmetric imaginary part, and since the latter has no isotropic component, it will be expected not to contribute to the final integrated decay rate.

In our partial evaluation of the matrix element, any factors related to the pion structure will be neglected. These factors are common to decays with and without Lorentz violation. Moreover, in the center of mass frame, the only vector or axial vector than can be associated with the parent particle is its momentum, $p_{\pi}=\left(m_{\pi}, 0,0,0\right)$. This simplifies the 
determination of the Lorentz-violating modifications considerably. However, the daughter particles do have spatial momenta, which will complicate the determination of the leptonic part of the matrix element somewhat. The momenta of the outgoing muon and antineutrino are $p_{\mu}=\left(\left[1+\xi^{2}\right] m_{\pi} / 2,\left[\left(1-\xi^{2}\right) m_{\pi} / 2\right] \hat{p}\right)$ and $p_{\nu}=\left(\left[1-\xi^{2}\right] m_{\pi} / 2,-\left[\left(1-\xi^{2}\right) m_{\pi} / 2\right] \hat{p}\right)$, where $\xi=m_{\mu} / m_{\pi}$ is the muon-pion mass ratio. (The labels $\mu$ and $\nu$ in these momenta denote the particle type and are not Lorentz indices; to avoid confusion, other letters will be used to denote Lorentz indices in expressions involving these vectors.) With no Lorentz violation in the purely leptonic sector, the expressions for $p_{\mu}$ and $p_{\nu}$ are conventional, and there are no changes to the kinematics of the decay.

The relevant part of the matrix element is given by

$$
\mathcal{M} \propto p_{\pi}^{\alpha}\left(\frac{g^{\alpha \beta}+\chi^{\alpha \beta}}{-m_{W}^{2}}\right) \bar{v}_{\nu}\left(p_{\nu}\right) \gamma_{\beta}\left(1-\gamma_{5}\right) u_{\mu}\left(p_{\mu}\right)
$$

Squaring this gives

$$
|\mathcal{M}|^{2} \propto \frac{m_{\pi}^{2}}{m_{W}^{4}}\left[\bar{v}_{\nu}\left(p_{\nu}\right)\left(\gamma_{0}+\chi^{0 \beta} \gamma_{\beta}\right)\left(1-\gamma_{5}\right) u_{\mu}\left(p_{\mu}\right) \bar{u}_{\mu}\left(p_{\mu}\right)\left(1+\gamma_{5}\right)\left(\gamma_{0}+\chi^{* 0 \gamma} \gamma_{\gamma}\right) v_{\mu}\left(p_{\mu}\right)\right]
$$

The sum over the outgoing spins can be evaluated using closure relations. Dropping the $m_{\pi}$ and $m_{W}$ prefactors, this gives

$$
\begin{aligned}
\sum_{s, s^{\prime}}|\mathcal{M}|^{2} \propto & \operatorname{tr}\left\{\not p_{\nu}\left(\gamma_{0}+\chi^{0 \beta} \gamma_{\beta}\right)\left(1-\gamma_{5}\right)\left(\not p_{\mu}+m_{\mu}\right)\left(1+\gamma_{5}\right)\left(\gamma_{0}+\chi^{* 0 \gamma} \gamma_{\gamma}\right)\right\} \\
= & 2\left(g^{0 \beta}+\chi^{0 \beta}\right)\left(g^{0 \gamma}+\chi^{* 0 \gamma}\right) \operatorname{tr}\left\{\not p_{\nu} \gamma_{\beta} \not p_{\mu} \gamma_{\gamma}-\not p_{\nu} \gamma_{\beta} \gamma_{5} \not p_{\mu} \gamma_{\gamma}\right\} \\
= & 8\left\{\left[2\left(p_{\mu}\right)_{0}\left(p_{\nu}\right)_{0}-p_{\mu} \cdot p_{\nu}\right]\right. \\
& +\left(\chi^{0 \beta}+\chi^{* 0 \beta}\right)\left[\left(p_{\mu}\right)_{0}\left(p_{\nu}\right)_{\beta}+\left(p_{\mu}\right)_{\beta}\left(p_{\nu}\right)_{0}-\left(p_{\mu} \cdot p_{\nu}\right) g_{0 \beta}\right] \\
& \left.+i g^{0 \gamma}\left(\chi^{0 \beta}-\chi^{* 0 \beta}\right) \epsilon_{\alpha \beta \delta \gamma}\left(p_{\nu}\right)^{\alpha}\left(p_{\mu}\right)^{\delta}\right\}
\end{aligned}
$$

The term proportional to $\epsilon_{\alpha \beta \delta \gamma}$ vanishes. While it was expected that the imaginary part of $\chi$ would not contribute to the total decay rate (because of its intrinsic anisotropy), it actually makes no leading-order contribution to the spin-summed $|\mathcal{M}|^{2}$, even prior to an angular integration over $\hat{p}$. The reason is that the $g^{0 \gamma} \epsilon_{\alpha \beta \delta \gamma}$ ensures that there is no contribution from the timelike components of $p_{\mu}$ and $p_{\nu}$; then since the spacelike components of these vectors are antiparallel, they also give an identically vanishing contribution when simultaneously contracted with the Levi-Civita tensor.

There is, however, a dependence on the imaginary part of $\chi$ in the spin structure of the cross section. Any such dependence on $\chi^{\mu \nu}-\chi^{* \mu \nu}$ must be related to muon spin correlations, since the neutrino has only a single interacting spin state. The $W$ boson only interacts with a single chirality state of the muon, but for a massive particle, $\gamma_{5}$ does not commute with the free-particle Hamiltonian, so both muon spin states are involved in the reaction. The expectation value of the spin provides an additional vector that may 
be contracted with the Levi-Civita tensor, along with $\chi^{\mu \nu}-\chi^{* \mu \nu}$ and $p_{\nu}$. The details of the dependence on the spin and the imaginary part of $\chi$ are discussed in the appendix.

The remaining anisotropic term in the spin-summed cross section does not vanish at this stage. Splitting the $\chi$ terms into separate spatial and temporal parts and using $p_{\mu} \cdot p_{\nu}=m_{\pi}^{2}\left(1-\xi^{2}\right) / 2$, the decay rate is proportional to

$$
\begin{aligned}
\sum_{s, s^{\prime}}|\mathcal{M}|^{2} & \propto\left(1+\chi^{00}+\chi^{* 00}\right) m_{\pi}^{2} \frac{\xi^{2}\left(1-\xi^{2}\right)}{2}-\left(\chi^{0 j}+\chi^{* 0 j}\right) \hat{p}_{j} m_{\pi}^{2} \frac{\xi^{2}\left(1-\xi^{2}\right)}{2} \\
& =\left(1+\chi^{0 \alpha}+\chi^{* 0 \alpha}\right) m_{\pi} \xi^{2}\left(p_{\nu}\right)_{\alpha} .
\end{aligned}
$$

This describes the anisotropy of the decay; the rate depends on the alignment of the outgoing particles with the axis described by $\Re\left\{\chi^{0 j}\right\}$. However, when integrated over all angles, the $\hat{p}$ term in (8) clearly gives a vanishing contribution. The net change to the pion decay rate is thus

$$
\Gamma=\Gamma_{0}\left(1+2 \chi^{00}\right)
$$

where $\Gamma_{0}$ is the rate in the absence of $\chi$.

The contribution of $\chi$ to the modified decay is simpler than the contribution of a similar coefficient $c_{L}$ in the left-handed fermion sector. The modification due to $c_{L}$ includes a dependence on the mass ratio $\xi$. However, it is not surprising that the effects of fermionic Lorentz violation are more intricate, because $c_{L}$ affects the phase space available to the outgoing fermions in a way that the weak parameter $\chi$ does not.

\section{Constraints on Weak Sector Lorentz Violation}

The result (10) depends only on the component of $\chi$ that is isotropic in the pion rest frame. However, pions with different velocities will see different values of $\chi^{00}$ and will decay at different rates. Thus there can actually be substantial anisotropy. Pions with different boosts relative to the laboratory will decay at different rates, and for a pion beam fixed to the Earth, the decay rate will exhibit sidereal oscillations.

To study this anisotropy, we must refer to the $\chi$ coefficients in a standard coordinate frame. The conventional choice for such a frame (in which experimental constraints on the SME parameters are usually expressed) uses Sun-centered celestial equatorial coordinates [42]. The $Z$-axis for these coordinates lies parallel to the Earth's axis; the $X$-axis indicates the direction of the vernal equinox point on the celestial sphere; and the $Y$-axis is set by the right hand rule. The capital indices $J$ and $K$ used below will refer to spatial coordinates in this specific frame. The choice of time coordinate is less essential; there is a standardized choice $T$, but it is usually advantageous to introduce an offset and use a local time coordinate $T_{\oplus}$, chosen to that the laboratory $y$-axis and the $Y$-axis lie parallel at $T_{\oplus}=0$. 
The isotropic $\chi^{00}$ in the pion rest frame is related to the tensor components in the Sun-centered frame by a boost. If the pion is moving ultrarelativistically (with a Lorentz factor $\gamma_{\pi} \gg 1$ ) along the direction $\hat{v}_{\pi}$, the boosted relationship is

$$
\chi^{00}=\gamma_{\pi}^{2}\left[\chi_{T T}+\chi_{(T J)}\left(\hat{v}_{\pi}\right)_{J}+\chi_{J K}\left(\hat{v}_{\pi}\right)_{J}\left(\hat{v}_{\pi}\right)_{K}\right],
$$

using the notation $\chi_{(\mu \nu)}=\chi_{\mu \nu}+\chi_{\nu \mu}$. The size of the effect in the pion rest frame depends on $\gamma_{\pi}^{2}$ and thus increases quite rapidly with the energy. This serves to enhance the sensitivity of measurements made with fast-moving pions. Note that, for an Earthbound laboratory and a beam of relativistic pions, there is no need to distinguish between the pion velocity relative to the lab and the velocity relative to the Sun-centered frame; any effects that distinguish between the two are suppressed by powers of $\gamma_{\pi}$. At highly relativistic energies, the decay products are also beamed into a narrow pencil of angles around the direction $\hat{v}_{\pi}$. This effectively washes out the anisotropy due to terms such as $\chi^{0 j}+\chi^{* 0 j}$.

Searches for evidence of Lorentz-violating neutrino oscillations in the NuMI beam using the MINOS near detector have already been done [43, 44]. The analyses looked for sidereal variations in the number of charged current events in the detector. However, as noted in [37, this can alternatively be interpreted as a search for directional variations in the neutrino beam strength. The signature of a direction-dependent pion decay rate would be a variation in the number of charged current events seen in the detector. This idea, along with the sidereal analysis from [43], has already been used to constrain the Lorentz violating $c_{L}$ coefficients for the second-generation leptons.

The techniques used here shall be quite similar, and much of the analysis is completely analogous. However, in both cases, there are additional complications, and the results should be seen as order of magnitude estimates. The primary concern is that, in particle physics experiments, the Lorentz violation may affect not just the rate at which a given interaction or decay occurs, but it may also change the efficiency with which the outgoing products are detected and identified. These kinds of detection issues are frequently sidestepped, as in [45], which considered Lorentz violation for $t$ quarks but did not address the way the forms of Lorentz violation involved would also affect the $b$ quark jets used to tag the $t$ events.

In the process being considered in this paper, the $\chi$ coefficients will generally modify not just $\Gamma$ but also the rate at which the NuMI beam neutrinos interact with the detector material. The charged current interactions that give rise to detectable signals are more complicated than the simple leptonic decay we have been discussing. However, there is good reason to believe that the interactions in the detector are less affected by the Lorentz violation than is the pion decay. The sensitivity of a weak process to the Lorentz violation coefficients goes as $E^{2}$, where $E$ is the lab frame energy. Since a decay neutrino carries off, on average, less than half of each pion's energy, the reactions at the detector are anticipated to be at least 4 time less sensitive to the observable components of $\chi$. Moreover, the effects of $\chi$ in the detector are generally expected to enhance, not 
diminish, the final observable. Because the boosted momenta that the $\chi^{\mu \nu}$ will ultimately be contracted with tend to point in roughly the same direction in the original pion decay and in the interaction at the detector, an enhancement of one reaction will generally be accompanied by an enhancement of the other as well. The net result is that the effect of neglecting modifications to the detector interactions should only be a modest loosening of the bounds that we shall be able to derive.

Of course, the intensity of the neutrino beam does not provide a direct measurement of $\Gamma / \Gamma_{0}$ either. What is effectively being measured is actually the total number of pions that manage to decay in the neutrino beam production region. The length $D$ of the decay pipe determines the magnitude of the variations in the neutrino beam strength. For example, if the decay pipe is very long, essentially all the pions will have time to decay, and the sensitivity to $\Gamma / \Gamma_{0}$ will be lost. Conversely, if the pie is very short, the number of decays will be directly proportional to $\Gamma / \Gamma_{0}$; however, the beam intensity will be low, and the detection statistics will suffer accordingly. In general, as $D$ grows, the fractional sensitivity to $\Gamma / \Gamma_{0}$ decreases, but the statistics improve.

Measured in the laboratory frame, the pion decay rate is $\Gamma / \gamma_{\pi}$, with the usual time dilation factor to account for the pions' relativistic motion; this factor is separate from those appearing in (11). Of pions moving with a speed $v_{\pi} \approx 1$ along the decay pipe of length $D$, a fraction $P(D)=1-e^{-\Gamma D / \gamma_{\pi}}$ have sufficient time to decay. Therefore, a fractional change $\Gamma / \Gamma_{0}$ in the instantaneous decay rate produces a fractional change in $P(D)$ of

$$
\left(\left.\frac{1}{P} \frac{d P}{d \Gamma}\right|_{\Gamma=\Gamma_{0}}\right) \Delta \Gamma=\left(\frac{\Delta \Gamma}{\Gamma_{0}}\right) \frac{\Gamma_{0} D / \gamma_{\pi}}{e^{\Gamma_{0} D / \gamma_{\pi}}-1} .
$$

In (12), a factor of $\Gamma_{0} / \Gamma_{0}$ has been inserted to make the expression into a product of two dimensionless quantities.

The decay pipe for the NuMI beam is $677 \mathrm{~m}$ long. This length was chosen to be comparable to the mean decay length for pions with $\mathrm{GeV}$ energies, which means the factor $\Gamma_{0} D / \gamma_{\pi}\left(e^{\Gamma_{0} D / \gamma_{\pi}}-1\right)$ appearing in (12) will be $\mathcal{O}(1)$. In fact, for a $6.0-\mathrm{GeV}$ pion (representative of the peak of the pion distribution), the value of the factor is 0.31 .

Following the reasoning from of [37], we can determine the manner in which the dependence of $\Gamma$ on $\chi$ will produce sidereal variations in the NuMI beam intensity. The MINOS near detector is located at colatitude $\varphi=42.18^{\circ}$ at Fermilab, and we will use spherical coordinates $(\theta, \phi)$ describing the angle between the beam direction and the local zenith direction $\left(\theta=93.27^{\circ}\right)$ and the azimuthal angle in the plane of the Earth's surface, measured starting eastward from south $\left(\phi=203.91^{\circ}\right)$. This makes the beam direction at a local sidereal time $T_{\oplus}=0$

$$
\begin{aligned}
\hat{v}_{\pi}= & N_{1} \hat{X}+N_{2} \hat{Y}+N_{3} \hat{Z} \\
= & (\cos \varphi \sin \theta \cos \phi+\sin \varphi \cos \theta) \hat{X}+(\sin \theta \sin \phi) \hat{Y} \\
& +(-\sin \varphi \sin \theta \cos \phi+\cos \varphi \cos \theta) \hat{Z}
\end{aligned}
$$




$$
=-0.715 \hat{X}-0.405 \hat{Y}+0.571 \hat{Z} \text {. }
$$

The revolution of the beam around the $\hat{Z}$-direction causes the the quantity $\chi^{00}$ upon with $\Gamma$ depends to vary as

$$
\chi^{00}=\gamma_{\pi}^{2}\left[\mathcal{A}_{0}+\mathcal{A}_{\omega} \cos \left(\omega_{\oplus} T_{\oplus}\right)+\mathcal{B}_{\omega} \sin \left(\omega_{\oplus} T_{\oplus}\right)+\mathcal{A}_{2 \omega} \cos \left(2 \omega_{\oplus} T_{\oplus}\right)+\mathcal{B}_{2 \omega} \sin \left(2 \omega_{\oplus} T_{\oplus}\right)\right],
$$

where $\omega_{\oplus}$ is the Earth's sidereal rotation frequency. The Fourier coefficients appearing in this expression are

$$
\begin{aligned}
\mathcal{A}_{0} & =\chi_{T T}+N_{3} \chi_{(T Z)}+N_{3}^{2} \chi_{Z Z}+\frac{1}{2}\left(1-N_{3}^{2}\right)\left[\chi_{X X}+\chi_{Y Y}\right] \\
\mathcal{A}_{\omega} & =N_{1} \chi_{(T X)}+N_{1} N_{3} \chi_{(X Z)}+N_{2} \chi_{(T Y)}+N_{2} N_{3} \chi_{(Y Z)} \\
\mathcal{B}_{\omega} & =-N_{2} \chi_{(T X)}-N_{2} N_{3} \chi_{(X Z)}+N_{1} \chi_{(T Y)}+N_{1} N_{3} \chi_{(Y Z)} \\
\mathcal{A}_{2 \omega} & =\frac{1}{2}\left(N_{1}^{2}-N_{2}^{2}\right) \chi_{-}+N_{1} N_{2} \chi_{(X Y)} \\
\mathcal{B}_{2 \omega} & =-N_{1} N_{2} \chi_{-}+\frac{1}{2}\left(N_{1}^{2}-N_{2}^{2}\right) \chi_{(X Y)},
\end{aligned}
$$

with $\chi_{-}=\chi_{X X}-\chi_{Y Y}$. (Since all the combinations appearing in the Fourier coefficients are symmetric, the expressions are all manifestly real.)

The neutrino beam intensity varies with the value of $\chi^{00}$ in the pion frame. The amplitudes of the beam strength oscillations are given by the $\mathcal{A}$ and $\mathcal{B}$ coefficients, times a common sensitivity factor

$$
\mathcal{S}=2 \gamma_{\pi}^{2}\left(\frac{\Gamma_{0} D / \gamma_{\pi}}{e^{\Gamma_{0} D / \gamma_{\pi}}-1}\right)=1.2 \times 10^{3}
$$

The factor of 2 in (22) comes directly from the expression for $\Gamma / \Gamma_{0}$; the $\gamma_{\pi}^{2}$ (which is the dominant contribution) derives from (16); and, as discussed above, the final factor in parentheses relates how a variation in $\Gamma / \Gamma_{0}$ affects total number of pion decays.

An analysis of $\chi$ based on the MINOS data mirrors the analyses from [43, 37]. There is no evidence (at a $3 \sigma$ level) for any of the oscillation amplitudes in the charged current event rate to be nonzero. The fractional level of statistical noise in the data was characterized by a $1 \sigma$ dispersion in the power values of $1.8 \times 10^{-2}$. We therefore assign $3 \sigma$ constraints on the quantities $\mathcal{S A}$ and $\mathcal{S B}$ at the $5.4 \times 10^{-2}$ level. This corresponds to constraints on the four separate oscillations amplitudes $\mathcal{A}_{\omega}, \mathcal{B}_{\omega}, \mathcal{A}_{2 \omega}$, and $\mathcal{B}_{2 \omega}$ of $4.5 \times 10^{-5}$. By assuming only one components of $\chi$ is nonzero at a time, the constraints on the $\mathcal{A}$ and $\mathcal{B}$ linear combinations can be translated into bounds on separate coefficients. The results are given in table 1,

\section{Discussion}

The constraints on the $\chi^{\mu \nu}$ coefficients are at the $10^{-4}$ level, representing an improvement over those discussed in [34], which were at the $10^{-3}$ level. The bounds cover both $\chi_{(J K)}$ 


\begin{tabular}{|c|c|}
\hline Coefficient & Bound \\
\hline$\left|\chi_{(T X)}\right|$ & $6.3 \times 10^{-5}$ \\
$\left|\chi_{(T Y)}\right|$ & $6.3 \times 10^{-5}$ \\
$\left|\chi_{-}\right|$ & $1.6 \times 10^{-4}$ \\
$\left|\chi_{(X Y)}\right|$ & $1.6 \times 10^{-4}$ \\
$\left|\chi_{(X Z)}\right|$ & $1.1 \times 10^{-4}$ \\
$\left|\chi_{(Y Z)}\right|$ & $1.1 \times 10^{-4}$ \\
\hline
\end{tabular}

Table 1: Constraints on the magnitudes of the individual $\chi$ coefficients in the Suncentered reference frame, assuming only a single coefficient is nonvanishing.

and $\chi_{(T J)}$ coefficients. However, none of these constraints are particularly tight, compared with bounds in other sectors of the SME. Moreover, these bounds are not on precisely the same $\chi$ parameters as might be measured in different processes, because of the momentum dependence in the $k_{W}$ term of (2). The present bounds are also comparable to those obtained on $\chi_{T T}$ in [38] by comparing the pion decay rate at different energy scales. Overall, there is ample room for improvement in this poorly constrained region of parameter space.

The bounds in table1 were derived under the assumption that only a single component of $\chi$ was nonvanishing. In reality, the MINOS data constrains the four linear combinations $\mathcal{A}_{\omega}, \mathcal{B}_{\omega}, \mathcal{A}_{2 \omega}$, and $\mathcal{B}_{2 \omega}$. By measuring sidereal changes in intensity using pion beams with a uniform energy but different terrestrial orientations, the sensitivity could be expanded to cover the six-parameter subspace considered spanned by $\chi_{T X}, \chi_{T Y}, \chi_{X Y}, \chi_{X Z}, \chi_{Y Z}$, and $\chi_{-}$. The remaining real coefficients, which determine $\mathcal{A}_{0}$, could be constrained by varying the pion energy. Varying the energy only (but not the beam direction) gives sensitivity to $\chi_{T T}$; this was essentially the approach taken in [38, in which isotropy was assumed. Varying both direction and energy provides sensitivities to the three remaining quantities $\chi_{T Z}, \chi_{Z Z}$, and $\chi_{X X}+\chi_{Y Y}$, which describe violations of isotropy and boost invariance in which the $Z$-axis represents the sole preferred direction. Finally, using different decaying meson species, with different rest frame energies, would make it possible to disentangle the $k_{W}$ coefficients from the others than make up the effecive quantity $\chi$.

There has actually been some confusion about how well the $k_{\phi \phi}, k_{\phi W}$, and $k_{W}$ that make up $\chi$ are known. In [39], much stronger bounds on some of the same SME coefficients were quoted. However, those bounds are not really accurate. There is a particular class of CPT-even SME coefficients - the $c$ coefficients for fermions, $k_{\phi \phi}$ in the Higgs sector, subsets of the $k_{F}$ and $k_{W}$ for electromagnetic and weak fields - that are observable only as differences between different sectors. Coordinate redefinitions can actually move the coefficients from one sector to another, but the differences between sectors are invariant under such transformations [46]. In some cases, such as analyses of the thresholds for ordinarily forbidden electron-photon processes like $\gamma \rightarrow e^{-}+e^{+}$, it is easy to identify 
unambiguously the specific difference involved. The threshold for the photon decay depends on the difference between photon-sector and electron-sector coefficients, because a comparison is effectively being made between the electron and photon dispersion relations.

However, in many low-energy experiments, precisely which sectors are being compared is not clear. In atomic clock experiments, the coefficients that are actively being studied are really being compared with some sort of aggregate coefficients for bulk matter. These aggregate coefficients are linear combinations of the coefficients for photons, electrons, protons, and neutrons (the latter two themselves being combinations of gluon and quark coefficients). In many cases, the comparison of different sectors is left implicit; it is assumed that there is only Lorentz violation for a single standard model field, and bounds are quoted for the corresponding coefficients in that sector.

However, only the coefficients for those sectors that are actually involved in a given process can be constrained by studying that process. In [39], the authors took constraints on various coefficients (which were not quoted as differences) as if they represented absolute bounds on the coefficients in a single sector. Then they used coordinate transformations to move the Lorentz violation to the weak sector and reinterpreted the experimental results as constraints on weak-sector Lorentz violation. Unfortunately, this methodology is not correct. It must be remembered that the experimental results really constrain only differences between coefficients in various sectors, and that the weak sector is not included as part of any of those differences. Since the differences between sectors are invariant under the redefinitions used in [39], the physical observables cannot be interpreted as depending on weak-sector SME coefficients.

The constraints in [39] on these CPT-even coefficients are not wholy meaningless, however. The weak sector does affect the physics of ordinary matter, through electroweak mixing and radiative mixing. The bounds quoted in [39] actually provide rough estimates of how large Lorentz violation in the weak sector can be without additional fine tuning. If Lorentz violation in the weak sector were much larger, there would need to be unnatural cancellations to prevent the large Lorentz violation from being transmitted to other sectors. However, naturalness, or the absence of fine tuning, is as aesthetic condition, not a rigorous one.

Considerations of naturalness and direct constraints via measurement therefore provide complementary approaches. Direct bounds on Lorentz violation, such as those discussed in this paper, are more rigorous. This makes them an important part of the developing analysis of the SME - espcially in the weak sector, which is, so far, quite poorly constrained.

\section{Acknowledgements}

The author is grateful to J. P. Noordmans, R. Timmermans, K. K. Vos, and H. W. Wilschut for helpful discussions. 


\section{Appendix: Dependence on the Imaginary Part of $\chi$}

We return now to the theoretical question of how spin measurements can add sensitivity to the imaginary part of the $\chi$ tensor describing weak and Higgs sector Lorentz violations. We shall replace the spin summed $u \bar{u}$ in (5) with the expression corresponding to specific outgoing spin state.

Throughout this paper, we have assumed that the inital pion was negatively charged, so that the daughter particles were $\mu^{-}$and $\bar{\nu}_{\mu}$. This choice was made simply for definiteness, and the result for the total decay rate $\Gamma$ is not affected by the choice. This is the case because the real part of $\chi$ is invariant under charge conjugation $(\mathrm{C})$. However, the imaginary part changes sign under $\mathrm{C}$, so the charges of the particles involved in the process are significant. In this appendix, we shall continue to assume that the parent particle is a $\pi^{-}$; however, if it were a $\pi^{+}$, all the effects of the imaginary part of $\chi$ would have their signs reversed.

Since the chirality matrix $\gamma_{5}$ plays a prominent role in the matrix element, it is convenient to use the Weyl representation of the Dirac matrices. With relativistic normalization, the spinor for a fermion of momentum $\vec{p}_{\mu}=p_{\mu 3} \hat{z}$ is

$$
u\left(p_{\mu}\right)=\left[\begin{array}{c}
\sqrt{E_{\mu}-p_{\mu 3} \sigma_{3}} \xi \\
\sqrt{E_{\mu}+p_{\mu 3} \sigma_{3}} \xi
\end{array}\right]=\left[\begin{array}{c}
\sqrt{p_{\mu} \cdot \sigma} \xi \\
\sqrt{p_{\mu} \cdot \bar{\sigma}} \xi
\end{array}\right],
$$

where $\sigma=(1, \vec{\sigma})$ and $\bar{\sigma}=(1,-\vec{\sigma})$. The two-spinor $\xi=\left[\xi_{1}, \xi_{2}\right]^{t}$ determines the spin state.

The quantity that appears in $|\mathcal{M}|^{2}$ is

$$
u \bar{u}=u u^{\dagger} \gamma_{0}=\left[\begin{array}{cc}
\sqrt{p_{\mu} \cdot \sigma} \xi \xi^{\dagger} \sqrt{p_{\mu} \cdot \bar{\sigma}} & \sqrt{p_{\mu} \cdot \sigma} \xi \xi^{\dagger} \sqrt{p_{\mu} \cdot \sigma} \\
\sqrt{p_{\mu} \cdot \bar{\sigma}} \xi \xi^{\dagger} \sqrt{p_{\mu} \cdot \bar{\sigma}} & \sqrt{p_{\mu} \cdot \bar{\sigma}} \xi \xi^{\dagger} \sqrt{p_{\mu} \cdot \sigma}
\end{array}\right] .
$$

This can be expanded in terms of the explicit spinor $\xi$ and momentum $p_{\mu}$. For example, the upper left block is

$$
\begin{aligned}
\sqrt{p_{\mu} \cdot \sigma \xi \xi^{\dagger} \sqrt{p_{\mu} \cdot \bar{\sigma}}} & =\left[\begin{array}{cc}
\sqrt{E_{\mu}-p_{\mu 3}} \xi_{1} \xi_{1}^{*} \sqrt{E_{\mu}+p_{\mu 3}} & \sqrt{E_{\mu}-p_{\mu 3}} \xi_{1} \xi_{2}^{*} \sqrt{E_{\mu}-p_{\mu 3}} \\
\sqrt{E_{\mu}+p_{\mu 3}} \xi_{2} \xi_{1}^{*} \sqrt{E_{\mu}+p_{\mu 3}} & \sqrt{E_{\mu}+p_{\mu 3}} \xi_{2} \xi_{2}^{*} \sqrt{E_{\mu}-p_{\mu 3}}
\end{array}\right] \\
& =\left[\begin{array}{cc}
m_{\mu} \xi_{1} \xi_{1}^{*} & \left(E_{\mu}-p_{\mu 3}\right) \xi_{1} \xi_{2}^{*} \\
\left(E_{\mu}+p_{\mu 3}\right) \xi_{2} \xi_{1}^{*} & m_{\mu} \xi_{2} \xi_{2}^{*}
\end{array}\right] .
\end{aligned}
$$

The other blocks reduce in a similar fashion.

It is also necessary to cast the quantities $\xi_{j} \xi_{k}^{*}$ in a more convenient form. The twospinor product $\xi \xi^{\dagger}$ may be concisely expressed in terms of the expectation value $\langle\vec{\sigma}\rangle=\xi^{\dagger} \vec{\sigma} \xi$ of the Pauli spin vector in the two-dimensional spinor space. It is important, however, that this is not the same as the expectation value of the full muon spin $\langle\vec{\Sigma}\rangle=\left(2 E_{\mu}\right)^{-1} u^{\dagger} \vec{\Sigma} u$. For the compoment of the spin along the direction of the motion, $\left\langle\Sigma_{3}\right\rangle=\left\langle\sigma_{3}\right\rangle$, since the helicity component of the spin commutes with a boost along the momentum direction. However, the expectation values of the transverse spin components are suppressed by 
relativistic effects, so that $\left\langle\Sigma_{j}\right\rangle=\frac{m_{\mu}}{E_{\mu}}\left\langle\sigma_{j}\right\rangle$ for $j=1$ or 2 . We shall return to this fact presently. However, for the moment we shall simply make use of the expression

$$
\xi \xi^{\dagger}=\left[\begin{array}{ll}
\xi_{1} \xi_{1}^{*} & \xi_{1} \xi_{2}^{*} \\
\xi_{2} \xi_{1}^{*} & \xi_{2} \xi_{2}^{*}
\end{array}\right]=\frac{1}{2}\left[\begin{array}{cc}
1+\left\langle\sigma_{3}\right\rangle & \left\langle\sigma_{-}\right\rangle \\
\left\langle\sigma_{+}\right\rangle & 1-\left\langle\sigma_{3}\right\rangle
\end{array}\right],
$$

with $\sigma_{ \pm}=\sigma_{1} \pm \sigma_{2}$.

The full $4 \times 4$ matrix $u \bar{u}$ is still rather cumbersome, and it can be expanded using the sixteen Dirac matrices as a basis. However, when this expression replaces $\left(\not p_{\mu}+m_{\mu}\right)$ in the trace (5), only those terms that are constructed from odd numbers of $\gamma$ matrices can contribute. This means the terms proportional to $\gamma_{\alpha}$ or $\gamma_{5} \gamma_{\alpha}$; equivalently, the only contributions come from the portion of $u \bar{u}$ that is block off-diagonal. (In fact, because of the way $u \bar{u}$ is sandwiched by chiral projectors, only a single off-diagonal block will ultimately contribute; however, it is computationally simpler to keep both off-diagonal blocks.) The block off-diagonal portion is given by (dropping the subscripts from $m_{\mu}, E_{\mu}$, and $p_{\mu 3}$ for brevity)

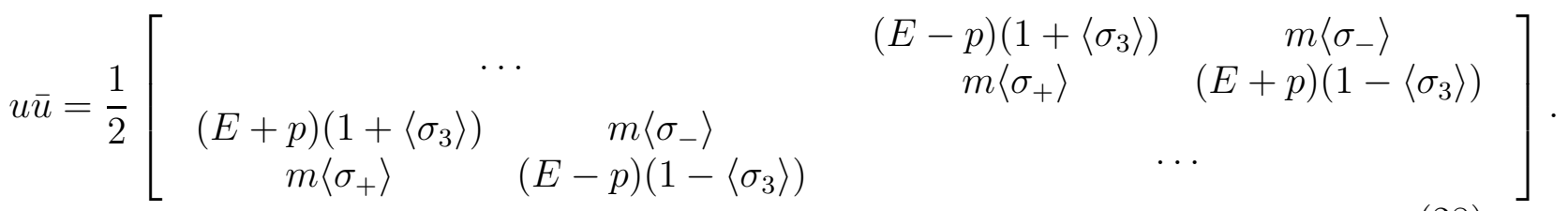

The expansion of this in terms of Dirac matrices is now straightforward. Leaving off the terms that will not contribute, we have

$$
u \bar{u}=\frac{1}{2}\left(E_{\mu} \gamma_{0}-p_{\mu 3} \gamma_{3}+p_{\mu 3}\left\langle\sigma_{3}\right\rangle \gamma_{5} \gamma_{0}-E_{\mu}\left\langle\sigma_{3}\right\rangle \gamma_{5} \gamma_{3}-m_{\mu}\left\langle\sigma_{1}\right\rangle \gamma_{5} \gamma_{1}-m_{\mu}\left\langle\sigma_{2}\right\rangle \gamma_{5} \gamma_{2}\right)+\ldots
$$

Note that the spin-independent term in (29) is simply the $\not \not_{\mu}$ that was already present in (5). However, there is also a new vector contracted with $\gamma_{5} \gamma_{\alpha}$. With $w=\left(\vec{p}_{\mu} \cdot\langle\vec{\Sigma}\rangle, E_{\mu}\langle\vec{\Sigma}\rangle\right)$, we have

$$
u \bar{u}=\frac{1}{2}\left(\not p_{\mu}+w^{\alpha} \gamma_{5} \gamma_{\alpha}\right)+\ldots
$$

So the analogue of (마) without the spin sum is

$$
|\mathcal{M}|^{2} \propto\left(g^{0 \beta}+\chi^{0 \beta}\right)\left(g^{0 \gamma}+\chi^{* 0 \gamma}\right) \operatorname{tr}\left\{\not p_{\nu} \gamma_{\beta}\left(\not p_{\mu}-\not \omega\right) \gamma_{\gamma}-\not p_{\nu} \gamma_{\beta} \gamma_{5}\left(\not p_{\mu}-\not \omega\right) \gamma_{\gamma}\right\}
$$

Whatever basis is chosen, the two muon spin states have opposite values of $\langle\vec{\Sigma}\rangle$, so summing over both of them sends the $w$-dependent terms to 0. For a nonrelativistic muon, there are substantial contributions from both helicity states; however, if the muon is ultrarelativistic $\left(m_{\mu} \rightarrow 0\right)$, the key quantity $p_{\mu}-w$ vanishes for the positive helicity states, and there are (as expected) no contributions from right-handed muons.

The $w$ vector influences both the $\chi^{\mu \nu}+\chi^{* \mu \nu}$ and $\chi^{\mu \nu}-\chi^{* \mu \nu}$ terms in $|\mathcal{M}|^{2}$. However, our main purpose here has been to obtain the dependence on the latter, since the imaginary 
part of $\chi$ is detectable only through spin correlations. The relevant term in the spindependent generalization of (17) is

$$
4 i g^{0 \gamma}\left(\chi^{0 \beta}-\chi^{* 0 \beta}\right) \epsilon_{\alpha \beta \delta \gamma}\left(p_{\nu}\right)^{\alpha}\left(-w^{\delta}\right)=8 E_{\mu} \vec{\chi} \cdot\left(\vec{p}_{\nu} \times\langle\vec{\Sigma}\rangle\right)
$$

where $\chi_{j}=\left(\chi^{0 j}-\chi^{* 0 j}\right) / 2 i$. Since the kinematics are not affected by the muon spin in any way, the anisotropic structure in (32) carries through to appear in the final spin-dependent differential decay rate. Only the spin components orthogonal to the momentum $\vec{p}_{\nu}=-\vec{p}_{\mu}$ contribute to the expression. Therefore, the effect does not grow with energy; the factor of $E_{\mu}$ only serves to balance the relativistic suppresion of the transverse part of $\langle\vec{\Sigma}\rangle$.

Measuring this term would be extremely challenging experimentally, but its existence is nonetheless theoretically interesting, and we have definitively demonstrated that the imaginary part of $\chi$ is, in principle, an observable quantity. This is in constrast with some other SME parameters, which may naively appear to be physically significant but, in fact, cannot be observed at all.

\section{References}

[1] D. Colladay, V. A. Kostelecký, Phys. Rev. D 55, 6760 (1997).

[2] D. Colladay, V. A. Kostelecký, Phys. Rev. D 58, 116002 (1998).

[3] R. Bluhm, V. A. Kostelecký, N. Russell, Phys. Rev. Lett. 79, 1432 (1997).

[4] G. Gabrielse, A. Khabbaz, D. S. Hall, C. Heimann, H. Kalinowsky, W. Jhe, Phys. Rev. Lett. 82, 3198 (1999).

[5] H. Dehmelt, R. Mittleman, R. S. Van Dyck, Jr., P. Schwinberg, Phys. Rev. Lett. 83, 4694 (1999).

[6] R. Bluhm, V. A. Kostelecký, N. Russell, Phys. Rev. Lett. 82, 2254 (1999).

[7] D. F. Phillips, M. A. Humphrey, E. M. Mattison, R. E. Stoner, R. F. C. Vessot, R. L. Walsworth, Phys. Rev. D 63, 111101(R) (2001).

[8] R. Bluhm, V. A. Kostelecký, C. D. Lane, Phys. Rev. Lett. 84, 1098 (2000).

[9] V. W. Hughes, et al., Phys. Rev. Lett. 87, 111804 (2001).

[10] B. R. Heckel, E. G. Adelberger, C. E. Cramer, T. S. Cook, S. Schlamminger, U. Schmidt, Phys. Rev. D 78, 092006 (2008).

[11] C. J. Berglund, L. R. Hunter, D. Krause, Jr., E. O. Prigge, M. S. Ronfeldt, S. K. Lamoreaux, Phys. Rev. Lett. 75, 1879 (1995). 
[12] V. A. Kostelecký, C. D. Lane, Phys. Rev. D 60, 116010 (1999).

[13] D. Bear, R. E. Stoner, R. L. Walsworth, V. A. Kostelecký, C. D. Lane, Phys. Rev. Lett. 85, 5038 (2000).

[14] P. Wolf, F. Chapelet, S. Bize, A. Clairon, Phys. Rev. Lett. 96, 060801 (2006).

[15] H. Müller, et al., Phys. Rev. Lett. 99, 050401 (2007).

[16] S. Herrmann, et al., Phys. Rev. D 80, 105011 (2009).

[17] Ch. Eisele, A. Yu. Nevsky, S. Schiller, Phys. Rev. Lett. 103, 090401 (2009).

[18] V. A. Kostelecký, Phys. Rev. D 61, 016002 (1999).

[19] Y. B. Hsiung, Nucl. Phys. Proc. Suppl. 86, 312 (2000).

[20] K. Abe et al. (Belle Collaboration), Phys. Rev. Lett. 86, 3228 (2001).

[21] J. M. Link et al., Phys. Lett. B 556, 7 (2003).

[22] B. Aubert et al. (BABAR Collaboration), Phys. Rev. Lett. 96, 251802 (2006).

[23] V. A. Kostelecký, M. Mewes, Phys. Rev. Lett. 97, 140401 (2006).

[24] V. A. Kostelecký, M. Mewes, Phys. Rev. Lett. 99, 011601 (2007).

[25] F. W. Stecker, S. L. Glashow, Astropart. Phys. 16, 97 (2001).

[26] T. Jacobson, S. Liberati, D. Mattingly, Nature 424, 1019 (2003).

[27] B. Altschul, Phys. Rev. Lett. 96, 201101 (2006).

[28] F. R. Klinkhamer, M. Risse, Phys. Rev. D 77, 016002 (2008); addendum Phys. Rev. D 77, 117901 (2008).

[29] J. B. R. Battat, J. F. Chandler, C. W. Stubbs, Phys. Rev. Lett. 99, 241103 (2007).

[30] H. Müller, S. W. Chiow, S. Herrmann, S. Chu, K.-Y. Chung, Phys. Rev. Lett. 100, 031101 (2008).

[31] V. A. Kostelecký, N. Russell, arXiv:0801.0287v6.

[32] T. Adam, et al. (OPERA collaboration), arXiv:1109.4897v1.

[33] J. P. Noordmans, H. W. Wilschut, R. G. E. Timmermans, Phys. Rev. C 87, 055502 (2013). 
[34] H. W. Wilschut, et al., arXiv:1303.6419,

[35] J. S. Diaz, V. A. Kostelecký, R. Lehnert, arXiv:1305.4636.

[36] B. Altschul, Phys. Rev. D 84, 091902(R) (2011).

[37] B. Altschul, Phys. Rev. D 87, 096004 (2013).

[38] H. B. Nielsen, I. Picek, Phys. Lett. B 114, 141 (1982).

[39] D. L. Anderson, M. Sher, I. Turan, Phys. Rev. D 70, 016001 (2004).

[40] R. Huerta, J. L. Lucio, Phys. Lett. B 131, 471 (1983).

[41] D. Colladay, V. A. Kostelecký, Phys. Lett. B 511209 (2001).

[42] R. Bluhm, V. A. Kostelecký, C. D. Lane, N. Russell, Phys. Rev. D 68, 125008 (2003).

[43] P. Adamson, et al. (MINOS Collaboration), Phys. Rev. Lett. 101, 151601 (2008).

[44] P. Adamson, et al. (MINOS Collaboration), Phys. Rev. D 85, 031101(R) (2012).

[45] V. M. Abazov, et al. (D0 Collaboration), Phys. Rev. Lett. 108, 261603 (2012).

[46] B. Altschul, Phys. Rev. Lett. 98, 041603 (2007). 